\title{
A note on the order derivatives of Kelvin functions
}

\author{
J. L. González-Santander \\ C/ Ovidi Montllor i Mengual 7, pta. 9. \\ 46017, Valencia, Spain.
}

\begin{abstract}
We calculate the derivative of the $\operatorname{ber}_{\nu}$, $\operatorname{bei}_{\nu}, \operatorname{ker}_{\nu}$, and $\mathrm{kei}_{\nu}$ functions with respect to the order $\nu$ in closed-form for $\nu \in \mathbb{R}$. Unlike the expressions found in the literature for order derivatives of the $\operatorname{ber}_{\nu}$ and $\mathrm{bei}_{\nu}$ functions, we provide much more simple expressions that are also applicable for negative integral order. The expressions for the order derivatives of the $\operatorname{ker}_{\nu}$ and $\mathrm{kei}_{\nu}$ functions seem to be novel. Also, as a by-product, we calculate some new integrals involving the $\operatorname{ber}_{\nu}$ and $\mathrm{bei}_{\nu}$ functions in closed-form. Finally, we include a simple derivation of some integral representations of the ber and bei functions.
\end{abstract}

Keywords: Kelvin functions, Bessel functions, generalized hypergeometric function, Meijer- $G$ function

Mathematics Subject Classification: 33C10, 33C20, 33E20

\section{Introduction}

In an investigation of the so-called skin effect in a wire carrying an alternating current, Lord Kelvin [1] introduced the ber and bei functions as the real and imaginary parts respectively of the regular solution of the differential equation

$$
x y^{\prime \prime}+y^{\prime}-i x y=0 .
$$

Lord Kelvin wrote this solution as

$$
I_{0}(\sqrt{i} x)=\operatorname{ber}(x)+i \text { bei }(x) .
$$

Since the main applications of the ber and bei functions comes from the analysis of the current distribution in circular conductors [2] where the argument $x$ represents the radial coordinate, we will consider hereafter $x \geq 0$. For other applications see [3, Chap. VIII-IX].

Whitehead 4 generalized the functions introduced by Lord Kelvin, denoting the real and imaginary parts of the solution of the differential equation

$$
x^{2} y^{\prime \prime}+x y^{\prime}-\left(\nu^{2}+i x^{2}\right) y=0,
$$


as (see also [5, Eqn. 10.61.1\&2])

$$
\begin{aligned}
\operatorname{ber}_{\nu}(x)+i \operatorname{bei}_{\nu}(x) & =e^{i \pi \nu} J_{\nu}\left(e^{-i \pi / 4} x\right), \\
\operatorname{ker}_{\nu}(x)+i \operatorname{kei}_{\nu}(x) & =e^{-i \pi \nu / 2} K_{\nu}\left(e^{i \pi / 4} x\right),
\end{aligned}
$$

where $\nu \in \mathbb{R}$. In the literature [5, Sect 10.61] we can find several properties of the Kelvin functions $\operatorname{ber}_{\nu}$, bei $_{\nu}, \operatorname{ker}_{\nu}$, and kei $_{\nu}$. However, regarding the integral representation of these functions, we have only found the results given in [6], which read as

$$
\begin{aligned}
& \operatorname{ber}_{\nu}(x \sqrt{2}) \\
= & \frac{1}{\pi} \int_{0}^{\pi}[\cos \pi \nu \cos (x \sin t-\nu t) \cosh (x \sin t) \\
& \quad-\sin \pi \nu \sin (x \sin t-\nu t) \sinh (x \sin t)] d t \\
& -\frac{\sin \pi \nu}{\pi} \int_{0}^{\infty} \exp (-\nu t-x \sinh t) \cos (x \sin t+\pi \nu) d t
\end{aligned}
$$

and

$$
\begin{aligned}
& \operatorname{bei}_{\nu}(x \sqrt{2}) \\
= & \frac{1}{\pi} \int_{0}^{\pi}[\cos \pi \nu \sin (x \sin t-\nu t) \sinh (x \sin t) \\
& +\sin \pi \nu \cos (x \sin t-\nu t) \cosh (x \sin t)] d t \\
& -\frac{\sin \pi \nu}{\pi} \int_{0}^{\infty} \exp (-\nu t-x \sinh t) \sin (x \sin t+\pi \nu) d t,
\end{aligned}
$$

In the Appendix, we obtain an alternative derivation quite simple for the integral representation of the ber and bei functions based on the idea given in [7]. From the integral representations (3) and (44), the derivatives with respect to the order are obtained in integral form as follows [6]:

$$
\begin{aligned}
& \frac{\partial \operatorname{ber}_{\nu}(x)}{\partial \nu} \\
= & \log \left(\frac{x}{2}\right) \operatorname{ber}_{\nu}(x)-\frac{3 \pi}{4} \operatorname{bei}_{\nu}(x) \\
& -\frac{x}{2 \sqrt{2}} \int_{0}^{1} u^{(\nu-1) / 2}[\gamma+\log (1-u)]\left[\operatorname{ber}_{\nu-1}(x \sqrt{u})+\operatorname{bei}_{\nu}(x \sqrt{u})\right] d u,
\end{aligned}
$$

and

$$
\begin{aligned}
& \frac{\partial \operatorname{bei}_{\nu}(x)}{\partial \nu} \\
= & \log \left(\frac{x}{2}\right) \operatorname{bei}_{\nu}(x)+\frac{3 \pi}{4} \operatorname{ber}_{\nu}(x) \\
& +\frac{x}{2 \sqrt{2}} \int_{0}^{1} u^{(\nu-1) / 2}[\gamma+\log (1-u)]\left[\operatorname{ber}_{\nu-1}(x \sqrt{u})-\operatorname{bei}_{\nu}(x \sqrt{u})\right] d u .
\end{aligned}
$$


Recently in [8], we find closed-form expressions for the order derivatives of the ber $_{\nu}$ and bei $_{\nu}$ functions, which read as

$$
\begin{aligned}
& \frac{\partial \operatorname{ber}_{\nu}(x)}{\partial \nu} \\
= & {\left[\log \left(\frac{x}{2}\right)-\psi(\nu)-\frac{1}{2 \nu}\right] \operatorname{ber}_{\nu}(x)-\frac{3 \pi}{4} \operatorname{bei}_{\nu}(x) } \\
& +\frac{\pi \csc \pi \nu}{2 \Gamma^{2}(\nu+1)}\left(\frac{x}{2}\right)^{2 \nu}\left[\sin \left(\frac{3 \pi \nu}{2}\right) \operatorname{bei}_{-\nu}(x)-\cos \left(\frac{3 \pi \nu}{2}\right) \operatorname{ber}_{-\nu}(x)\right] c(\nu, x, 0) \\
& +\frac{\pi \nu \csc \pi \nu}{\Gamma^{2}(\nu+2)}\left(\frac{x}{2}\right)^{2 \nu+2}\left[\cos \left(\frac{3 \pi \nu}{2}\right) \operatorname{bei}_{-\nu}(x)+\sin \left(\frac{3 \pi \nu}{2}\right) \operatorname{ber}_{-\nu}(x)\right] c(\nu, x, 1) \\
& -\frac{x^{2}}{4\left(1-\nu^{2}\right)}\left[\operatorname{bei}_{\nu}(x) d(\nu, x, 0)+\frac{3 x^{2}}{8\left(4-\nu^{2}\right)} \operatorname{ber}_{\nu}(x) d(\nu, x, 1)\right],
\end{aligned}
$$

and

$$
\begin{aligned}
& \frac{\partial \operatorname{bei}_{\nu}(x)}{\partial \nu} \\
= & {\left[\log \left(\frac{x}{2}\right)-\psi(\nu)-\frac{1}{2 \nu}\right] \operatorname{bei}_{\nu}(x)+\frac{3 \pi}{4} \operatorname{ber}_{\nu}(x) } \\
& -\frac{\pi \csc \pi \nu}{2 \Gamma^{2}(\nu+1)}\left(\frac{x}{2}\right)^{2 \nu}\left[\cos \left(\frac{3 \pi \nu}{2}\right) \operatorname{bei}_{-\nu}(x)+\sin \left(\frac{3 \pi \nu}{2}\right) \operatorname{ber}_{-\nu}(x)\right] c(\nu, x, 0) \\
& -\frac{\pi \nu \csc \pi \nu}{\Gamma^{2}(\nu+2)}\left(\frac{x}{2}\right)^{2 \nu+2}\left[\cos \left(\frac{3 \pi \nu}{2}\right) \operatorname{ber}_{-\nu}(x)-\sin \left(\frac{3 \pi \nu}{2}\right) \operatorname{bei}_{-\nu}(x)\right] c(\nu, x, 1) \\
& +\frac{x^{2}}{4\left(1-\nu^{2}\right)}\left[\operatorname{ber}_{\nu}(x) d(\nu, x, 0)-\frac{3 x^{2}}{8\left(4-\nu^{2}\right)} \operatorname{bei}_{\nu}(x) d(\nu, x, 1)\right],
\end{aligned}
$$

and where the following functions have been defined:

$$
\begin{aligned}
& c(\nu, x, a) \\
= & { }_{3} F_{6}\left(\begin{array}{c|c}
\frac{2 \nu+a+1}{4}, \frac{2 \nu+3}{4}, \frac{2 \nu+5 a}{4} \\
a+\frac{1}{2}, \frac{\nu+a+1}{2}, \frac{\nu+a}{2}+1, \frac{\nu+a}{2}+1, \nu+\frac{a+1}{2}, \nu+1+\frac{a}{2}
\end{array} \mid-\frac{x^{4}}{16}\right),
\end{aligned}
$$

and

$$
\begin{aligned}
& d(\nu, x, a) \\
= & { }_{4} F_{7}\left(a+\frac{1}{2}, \frac{a+3}{2}, \frac{a+3}{2}, \frac{a+1}{2}, \frac{a+1}{2}, \frac{2 a+3}{4}, \frac{2 a+5}{4}, \frac{\nu+a+3}{2}, \frac{a-\nu}{2}+1, \frac{a-\nu+3}{2} \mid-\frac{x^{4}}{16}\right) .
\end{aligned}
$$

It is worth noting that (7) and (8) cannot be applicable for integral order, i.e. $\nu=n \in \mathbb{Z}$. Nevertheless, for $n \geq 0$, we find in the literature the following expressions [9, Eqn. 1.17.2(1)\&(2)]:

$$
\begin{aligned}
& \left.\frac{\partial \operatorname{ber}_{\nu}(x)}{\partial \nu}\right|_{\nu=n}=-\frac{\pi}{2} \operatorname{bei}_{n}(x)-\operatorname{ker}_{n}(x) \\
& +\frac{n !}{2} \sum_{k=0}^{n-1} \frac{(z / 2)^{k-n}}{k !(n-k)}\left[\cos \left(\frac{5(k-n) \pi}{4}\right) \operatorname{ber}_{k}(x)+\sin \left(\frac{5(k-n) \pi}{4}\right) \operatorname{bei}_{k}(x)\right]
\end{aligned}
$$


and

$$
\begin{aligned}
& \left.\frac{\partial \operatorname{bei}_{\nu}(x)}{\partial \nu}\right|_{\nu=n}=\frac{\pi}{2} \operatorname{ber}_{n}(x)-\operatorname{kei}_{n}(x) \\
& +\frac{n !}{2} \sum_{k=0}^{n-1} \frac{(z / 2)^{k-n}}{k !(n-k)}\left[\cos \left(\frac{5(k-n) \pi}{4}\right) \operatorname{bei}_{k}(x)-\sin \left(\frac{5(k-n) \pi}{4}\right) \operatorname{ber}_{k}(x)\right]
\end{aligned}
$$

Also, for the $\operatorname{ker}_{\nu}$ and $\operatorname{kei}_{\nu}$ functions, we have for $n \geq 0$, Eqn. 1.17.2(5)\&(6)]:

$$
\begin{aligned}
& \left.\frac{\partial \operatorname{ker}_{\nu}(x)}{\partial \nu}\right|_{\nu=n}=\frac{\pi}{2} \operatorname{kei}_{n}(x) \\
& +\frac{n !}{2} \sum_{k=0}^{n-1} \frac{(z / 2)^{k-n}}{k !(n-k)}\left[\cos \left(\frac{3(k-n) \pi}{4}\right) \operatorname{ker}_{k}(x)-\sin \left(\frac{3(k-n) \pi}{4}\right) \operatorname{kei}_{k}(x)\right]
\end{aligned}
$$

and

$$
\begin{aligned}
& \left.\frac{\partial \operatorname{kei}_{\nu}(x)}{\partial \nu}\right|_{\nu=n}=-\frac{\pi}{2} \operatorname{ker}_{n}(x) \\
& +\frac{n !}{2} \sum_{k=0}^{n-1} \frac{(z / 2)^{k-n}}{k !(n-k)}\left[\sin \left(\frac{3(k-n) \pi}{4}\right) \operatorname{ker}_{k}(x)+\cos \left(\frac{3(k-n) \pi}{4}\right) \operatorname{kei}_{k}(x)\right]
\end{aligned}
$$

Nonetheless, integral representations and explicit closed-form expressions for the order derivatives of the $\operatorname{ker}_{\nu}$ and $\mathrm{kei}_{\nu}$ functions for non-integral order are apparently absent in the literature. Therefore, the main scope of this paper is to obtain alternative closed-form expressions for the order derivatives of the $\operatorname{ber}_{\nu}$ and bei $_{\nu}$ functions, and new ones for the order derivatives of the $\operatorname{ker}_{\nu}$ and $\mathrm{kei}_{\nu}$ functions for arbitrary real order, $\nu \in \mathbb{R}$. From these results, and taking into account the integral representations given in (5) and (6), we will obtain the calculation of some integrals involving the Kelvin functions $\operatorname{ber}_{\nu}$ and bei $_{\nu}$.

This paper is organized as follows. Section 2 is devoted to the calculation of the derivatives of the Kelvin functions with respect to the order. First, we calculate the corresponding order derivatives for non-negative order, and then, using reflection formulas, we calculate the case of negative order. In Section 3, we calculate some integrals involving the $\operatorname{ber}_{\nu}$ and bei $_{\nu}$ functions, which do not seem to be reported in the literature. Finally, we collect the conclusions in Section 4 . Also, we provide an alternative derivation for the integral representation of the ber and bei functions in the Appendix.

\section{Order derivatives of Kelvin functions}

According to the definitions given in (11) and (2), $\forall x, \nu \geq 0$ we have that

$$
\begin{aligned}
\operatorname{ber}_{\nu}(x) & =\operatorname{Re}\left[e^{i \pi \nu} J_{\nu}\left(e^{-i \pi / 4} x\right)\right], \\
\operatorname{bei}_{\nu}(x) & =\operatorname{Im}\left[e^{i \pi \nu} J_{\nu}\left(e^{-i \pi / 4} x\right)\right],
\end{aligned}
$$


and

$$
\begin{aligned}
\operatorname{ker}_{\nu}(x) & =\operatorname{Re}\left[e^{-i \pi \nu / 2} K_{\nu}\left(e^{i \pi / 4} x\right)\right], \\
\operatorname{kei}_{\nu}(x) & =\operatorname{Im}\left[e^{-i \pi \nu / 2} K_{\nu}\left(e^{i \pi / 4} x\right)\right] .
\end{aligned}
$$

We can rewrite (13)-(16), using the fact that

$$
\begin{aligned}
& \operatorname{Re} z=\frac{z+\bar{z}}{2}, \\
& \operatorname{Im} z=\frac{z-\bar{z}}{2 i},
\end{aligned}
$$

and the properties $\forall \nu \in \mathbb{R}$ [5, Eqn. 10.11.8\&10.34.7]

$$
\begin{aligned}
\overline{J_{\nu}(z)} & =J_{\nu}(\bar{z}), \\
\overline{K_{\nu}(z)} & =K_{\nu}(\bar{z}),
\end{aligned}
$$

thereby

$$
\begin{aligned}
\operatorname{ber}_{\nu}(x) & =\frac{1}{2}\left[e^{i \pi \nu} J_{\nu}\left(e^{-i \pi / 4} x\right)+e^{-i \pi \nu} J_{\nu}\left(e^{i \pi / 4} x\right)\right] \\
\operatorname{bei}_{\nu}(x) & =\frac{i}{2}\left[e^{-i \pi \nu} J_{\nu}\left(e^{i \pi / 4} x\right)-e^{i \pi \nu} J_{\nu}\left(e^{-i \pi / 4} x\right)\right]
\end{aligned}
$$

and

$$
\begin{aligned}
\operatorname{ker}_{\nu}(x) & =\frac{1}{2}\left[e^{i \pi \nu / 2} K_{\nu}\left(e^{i \pi / 4} x\right)+e^{i \pi \nu / 2} K_{\nu}\left(e^{-i \pi / 4} x\right)\right] \\
\operatorname{kei}_{\nu}(x) & =\frac{i}{2}\left[e^{i \pi \nu / 2} K_{\nu}\left(e^{-i \pi / 4} x\right)-e^{-i \pi \nu / 2} K_{\nu}\left(e^{i \pi / 4} x\right)\right] .
\end{aligned}
$$

Thus, taking into account (13)-(18), the derivative of the Kelvin functions with respect to the order can be expressed as follows:

Theorem $1 \forall x, \nu \geq 0$, the order derivatives of the Kevin functions are

$$
\begin{aligned}
& \frac{\partial \operatorname{ber}_{\nu}(x)}{\partial \nu}=\operatorname{Re}\left[e^{i \pi \nu} \frac{\partial J_{\nu}}{\partial \nu}\left(e^{-i \pi / 4} x\right)\right]-\pi \operatorname{bei}_{\nu}(x), \\
& \frac{\partial \operatorname{bei}_{\nu}(x)}{\partial \nu}=\operatorname{Im}\left[e^{i \pi \nu} \frac{\partial J_{\nu}}{\partial \nu}\left(e^{-i \pi / 4} x\right)\right]+\pi \operatorname{ber}_{\nu}(x),
\end{aligned}
$$

and

$$
\begin{aligned}
& \frac{\partial \operatorname{ker}_{\nu}(x)}{\partial \nu}=\operatorname{Re}\left[e^{-i \pi \nu / 2} \frac{\partial K_{\nu}}{\partial \nu}\left(e^{i \pi / 4} x\right)\right]+\frac{\pi}{2} \operatorname{kei}_{\nu}(x), \\
& \frac{\partial \operatorname{kei}_{\nu}(x)}{\partial \nu}=\operatorname{Im}\left[e^{-i \pi \nu / 2} \frac{\partial K_{\nu}}{\partial \nu}\left(e^{i \pi / 4} x\right)\right]-\frac{\pi}{2} \operatorname{ker}_{\nu}(x) .
\end{aligned}
$$


For negative order $\nu$, we can use the following reflection formulas [5, Eqn. 10.61.6\&7]:

$$
\begin{aligned}
\operatorname{ber}_{-\nu}(x) & =\cos \pi \nu \operatorname{ber}_{\nu}(x)+\sin \pi \nu \operatorname{bei}_{\nu}(x)+\frac{2}{\pi} \sin \pi \nu \operatorname{ker}_{\nu}(x), \\
\operatorname{bei}_{-\nu}(x) & =-\sin \pi \nu \operatorname{ber}_{\nu}(x)+\cos \pi \nu \operatorname{bei}_{\nu}(x)+\frac{2}{\pi} \sin \pi \nu \operatorname{kei}_{\nu}(x),
\end{aligned}
$$

and

$$
\begin{aligned}
\operatorname{ker}_{-\nu}(x) & =\cos \pi \nu \operatorname{ker}_{\nu}(x)-\sin \pi \nu \operatorname{kei}_{\nu}(x), \\
\operatorname{kei}_{-\nu}(x) & =\sin \pi \nu \operatorname{ker}_{\nu}(x)+\cos \pi \nu \operatorname{kei}_{\nu}(x) .
\end{aligned}
$$

Theorem $2 \forall x, \nu>0$, the following order derivatives hold true:

$$
\begin{aligned}
& \frac{\partial \operatorname{ber}_{-\nu}(x)}{\partial \nu} \\
= & -\operatorname{Re}\left[e^{i \pi \nu / 2}\left\{\left(e^{-i \pi \nu}+\cos \pi \nu\right) K_{\nu}\left(e^{i \pi / 4} x\right)+\frac{2}{\pi} \sin \pi \nu \frac{\partial K_{\nu}}{\partial \nu}\left(e^{i \pi / 4} x\right)\right\}\right. \\
& \left.+\frac{\partial J_{\nu}}{\partial \nu}\left(e^{-i \pi / 4} x\right)\right],
\end{aligned}
$$

and

$$
\begin{aligned}
& \frac{\partial \operatorname{ber}_{-\nu}(x)}{\partial \nu} \\
= & -\operatorname{Im}\left[e^{i \pi \nu / 2}\left\{\left(e^{-i \pi \nu}+\cos \pi \nu\right) K_{\nu}\left(e^{i \pi / 4} x\right)+\frac{2}{\pi} \sin \pi \nu \frac{\partial K_{\nu}}{\partial \nu}\left(e^{i \pi / 4} x\right)\right\}\right. \\
& \left.+\frac{\partial J_{\nu}}{\partial \nu}\left(e^{-i \pi / 4} x\right)\right] .
\end{aligned}
$$

Proof. Performing the order derivative in (23) and taking into account (19) and (20), we arrive at

$$
\begin{aligned}
& -\frac{\partial \operatorname{ber}_{-\nu}(x)}{\partial \nu} \\
= & \sin \pi \nu \operatorname{Im}\left[e^{i \pi \nu} \frac{\partial J_{\nu}}{\partial \nu}\left(e^{-i \pi / 4} x\right)\right]+\cos \pi \nu \operatorname{Re}\left[e^{i \pi \nu} \frac{\partial J_{\nu}}{\partial \nu}\left(e^{-i \pi / 4} x\right)\right] \\
& +2 \cos \pi \nu \operatorname{ker}_{\nu}(x)+\frac{2}{\pi} \sin \pi \nu \frac{\partial \operatorname{ker}_{\nu}(x)}{\partial \nu} .
\end{aligned}
$$

Consider now (21) and the property

$$
\cos \pi \nu \operatorname{Re} z \pm \sin \pi \nu \operatorname{Im} z=\operatorname{Re}\left(e^{\mp i \pi \nu} z\right),
$$


to rewrite (29) as

$$
\begin{aligned}
-\frac{\partial \operatorname{ber}_{-\nu}(x)}{\partial \nu}= & \operatorname{Re}\left[\frac{\partial J_{\nu}}{\partial \nu}\left(e^{-i \pi / 4} x\right)\right]+2 \cos \pi \nu \operatorname{ker}_{\nu}(x) \\
& +\sin \pi \nu \operatorname{kei}_{\nu}(x)+\frac{2}{\pi} \sin \pi \nu \operatorname{Re}\left[e^{-i \pi \nu / 2} \frac{\partial K_{\nu}}{\partial \nu}\left(e^{i \pi / 4} x\right)\right] .
\end{aligned}
$$

Finally, substitute (15) and (16), and apply again (30) to arrive at (27). We can perform a similar proof for (28), applying the property

$$
\cos \pi \nu \operatorname{Im} z \pm \sin \pi \nu \operatorname{Re} z=\operatorname{Im}\left(e^{ \pm i \pi \nu} z\right) .
$$

Theorem $3 \forall x, \nu>0$, the following order derivatives hold true:

$$
\frac{\partial \operatorname{ker}_{-\nu}(x)}{\partial \nu}=\frac{\pi}{2} \operatorname{Im}\left[e^{i \pi \nu / 2} K_{\nu}\left(e^{i \pi / 4} x\right)\right]-\operatorname{Re}\left[e^{i \pi \nu / 2} \frac{\partial K_{\nu}}{\partial \nu}\left(e^{i \pi / 4} x\right)\right]
$$

and

$$
\frac{\partial \operatorname{kei}_{-\nu}(x)}{\partial \nu}=-\frac{\pi}{2} \operatorname{Re}\left[e^{i \pi \nu / 2} K_{\nu}\left(e^{i \pi / 4} x\right)\right]-\operatorname{Im}\left[e^{i \pi \nu / 2} \frac{\partial K_{\nu}}{\partial \nu}\left(e^{i \pi / 4} x\right)\right] .
$$

Proof. Perform in (25) the derivative with respect to the order, taking into account (21) and (22), to arrive at

$$
\begin{aligned}
-\frac{\partial \operatorname{ker}_{-\nu}(x)}{\partial \nu}= & -\frac{\pi}{2} \sin \pi \nu \operatorname{ker}_{\nu}(x)+\cos \pi \nu \operatorname{Re}\left[e^{-i \pi \nu / 2} \frac{\partial K_{\nu}}{\partial \nu}\left(e^{i \pi / 4} x\right)\right] \\
& -\frac{\pi}{2} \cos \pi \nu \operatorname{kei}_{\nu}(x)-\sin \pi \nu \operatorname{Im}\left[e^{-i \pi \nu / 2} \frac{\partial K_{\nu}}{\partial \nu}\left(e^{i \pi / 4} x\right)\right] .
\end{aligned}
$$

Substitute now (15) and (16) and apply the properties (15) and (31) to obtain (32). Similarly, we can derive (33).

Therefore, according to the results of the above theorems, knowing $\partial J_{\nu} / \partial \nu$ and $\partial K_{\nu} / \partial \nu$ in closed-form, we can express the order derivatives of the Kelvin functions in closed-form as well. Recently in [10], the order derivative of the Bessel function of the first kind $\forall \nu>0, z \in \mathbb{C}, z \neq 0$, is expressed in closed-form as follows:

$$
\begin{aligned}
& \frac{\partial J_{\nu}(z)}{\partial \nu} \\
= & \frac{-\pi J_{-\nu}(z) \csc \pi \nu}{2 \Gamma^{2}(\nu+1)}\left(\frac{z}{2}\right)^{2 \nu}{ }_{2} F_{3}\left(\begin{array}{c}
\nu, \nu+\frac{1}{2} \\
\nu+1, \nu+1,2 \nu+1
\end{array} \mid-z^{2}\right) \\
& -J_{\nu}(z)\left[\frac{z^{2}}{4\left(1-\nu^{2}\right)}{ }_{3} F_{4}\left(\begin{array}{c}
1,1, \frac{3}{2} \\
2,2,2-\nu, 2+\nu
\end{array} \mid-z^{2}\right)\right. \\
& \left.\quad+\log \left(\frac{2}{z}\right)+\frac{1}{2 \nu}+\psi(\nu)\right] .
\end{aligned}
$$


However, (34) cannot be used for non-negative integral orders, i.e. $\quad \nu=$ $0,1,2, \ldots$. A way to avoid this inconvenient is to express the derivative of $J_{\nu}(z)$ with respect to the order using the Meijer- $G$ function [10],

$$
\begin{aligned}
\frac{\partial J_{\nu}(z)}{\partial \nu}= & \frac{\pi}{2}\left[Y_{\nu}(z) \frac{(z / 2)^{2 \nu}}{\Gamma^{2}(\nu+1)}{ }_{2} F_{3}\left(\begin{array}{c}
\nu, 1 / 2+\nu \\
2 \nu+1, \nu+1, \nu+1
\end{array} \mid-z^{2}\right)\right. \\
& \left.-\frac{\nu J_{\nu}(z)}{\sqrt{\pi}} G_{2,4}^{3,0}\left(\begin{array}{l|c}
z^{2} & 1 / 2,1 \\
0,0, \nu,-\nu
\end{array}\right)\right], \quad \nu \geq 0, \operatorname{Re} z>0
\end{aligned}
$$

Similarly, for the order derivative of the Macdonald function, $\forall \nu>0, z \in \mathbb{C}$, $z \neq 0$, we have [10],

$$
\begin{aligned}
& \frac{\partial K_{\nu}(z)}{\partial \nu} \\
= & \frac{\pi}{2} \csc \pi \nu\left\{\pi \cot \pi \nu I_{\nu}(z)-\left[I_{\nu}(z)+I_{-\nu}(z)\right]\right. \\
& {\left.\left[\frac{z^{2}}{4\left(1-\nu^{2}\right)}{ }_{3} F_{4}\left(\begin{array}{c}
1,1, \frac{3}{2} \\
2,2,2-\nu, 2+\nu
\end{array} \mid z^{2}\right)+\log \left(\frac{z}{2}\right)-\psi(\nu)-\frac{1}{2 \nu}\right]\right\} } \\
+ & \frac{1}{4}\left\{I_{-\nu}(z) \Gamma^{2}(\nu)\left(\frac{z}{2}\right)^{2 \nu}{ }_{2} F_{3}\left(\begin{array}{c}
1+\frac{1}{2}+\nu \\
1+\nu, 1+\nu, 2 \nu
\end{array} \mid z^{2}\right)\right. \\
& \left.-I_{\nu}(z) \Gamma^{2}(-\nu)\left(\frac{z}{2}\right)^{-2 \nu}{ }_{2} F_{3}\left(\begin{array}{c}
\nu, \frac{1}{2}-\nu \\
1-\nu, 1-\nu, 1-2 \nu
\end{array} \mid z^{2}\right)\right\}
\end{aligned}
$$

Again, (36) cannot be used for non-negative integral orders, as well as for non-negative half-integral orders, i.e. $\nu=0,1 / 2,1,3 / 2, \ldots$ In these cases, we can employ the following representation, which uses Meijer- $G$ functions:

$$
\begin{aligned}
& \frac{\partial K_{\nu}(z)}{\partial \nu} \\
= & \frac{\nu}{2}\left[\frac{K_{\nu}(z)}{\sqrt{\pi}} G_{2,4}^{3,1}\left(z^{2} \mid \begin{array}{c}
1 / 2,1 \\
0,0, \nu,-\nu
\end{array}\right)-\sqrt{\pi} I_{\nu}(z) G_{2,4}^{4,0}\left(\begin{array}{l|l}
z^{2} & 1 / 2,1 \\
0,0, \nu,-\nu
\end{array}\right)\right] \\
& \nu \geq 0, \operatorname{Re} z>0 .
\end{aligned}
$$

It is worth noting that the numerical evaluation of (34) and (36) is $\approx 10$ times faster than (35) and (37), respectively.

\section{Application to the calculation of some inte- grals}

Comparing the integral representations given in the Introduction to the results given in Section 2, we can calculate some integrals involving Kelvin functions that do not seem to be reported in the literature. 
Theorem 4 The following integrals hold true:

$$
\begin{aligned}
& \int_{0}^{1} u^{\nu+1} \log \left(1-u^{2}\right) f_{\nu}(x u) d u \\
= & \frac{1}{\sqrt{2} x}\left\{\left[\frac{\pi}{4}+\log \left(\frac{x}{2}\right)+\gamma\right] f_{\nu+1}(x)\right. \\
& \left. \pm\left[\frac{\pi}{4}-\log \left(\frac{x}{2}\right)-\gamma\right] g_{\nu+1}(x)+\sqrt{2} \operatorname{Re}\left[e^{i \pi(\nu \pm 1 / 4)} \frac{\partial J_{\nu}}{\partial \nu}\left(e^{-i \pi / 4} x\right)\right]\right\},
\end{aligned}
$$

where $f_{\nu}$ and $g_{\nu}$ denote the ordered pair ber $_{\nu}$ and bei $_{\nu}$.

Proof. If we equate (5) to (19), and then we shift the order $\nu \rightarrow \nu+1$, and we change the integration variable $u \rightarrow u^{2}$, we obtain

$$
\begin{aligned}
& \int_{0}^{1} u^{\nu / 2}\left[\gamma+\log \left(1-u^{2}\right)\right]\left[\operatorname{ber}_{\nu}(x u)+\operatorname{bei}_{\nu}(x u)\right] d u \\
= & \frac{\sqrt{2}}{x}\left\{\frac{\pi}{4} \operatorname{bei}_{\nu+1}(x)+\log \left(\frac{x}{2}\right) \operatorname{ber}_{\nu+1}(x)-\operatorname{Re}\left[e^{i \pi(\nu+1)} \frac{\partial J_{\nu}}{\partial \nu}\left(e^{-i \pi / 4} x\right)\right]\right\} .
\end{aligned}
$$

Similarly, from (6) and (20), we arrive at

$$
\begin{aligned}
& \int_{0}^{1} u^{\nu / 2}\left[\gamma+\log \left(1-u^{2}\right)\right]\left[\operatorname{ber}_{\nu}(x u)+\operatorname{bei}_{\nu}(x u)\right] d u \\
= & \frac{\sqrt{2}}{x}\left\{\frac{\pi}{4} \operatorname{ber}_{\nu+1}(x)-\log \left(\frac{x}{2}\right) \operatorname{bei}_{\nu+1}(x)+\operatorname{Im}\left[e^{i \pi(\nu+1)} \frac{\partial J_{\nu}}{\partial \nu}\left(e^{-i \pi / 4} x\right)\right]\right\} .
\end{aligned}
$$

Summing up (39) and (40), and taking into account the property

$$
\operatorname{Im} z-\operatorname{Re} z=\sqrt{2} \operatorname{Re}\left(e^{-i 3 \pi / 4} z\right),
$$

we have

$$
\begin{aligned}
& \int_{0}^{1} u^{\nu / 2}\left[\gamma+\log \left(1-u^{2}\right)\right] \operatorname{ber}_{\nu}(x u) d u \\
=\frac{1}{\sqrt{2} x} & \left\{\left[\frac{\pi}{4}+\log \left(\frac{x}{2}\right)\right] \operatorname{ber}_{\nu+1}(x)+\left[\frac{\pi}{4}-\log \left(\frac{x}{2}\right)\right] \operatorname{bei}_{\nu+1}(x)\right. \\
& \left.+\sqrt{2} \operatorname{Re}\left[e^{i \pi(\nu+1 / 4)} \frac{\partial J_{\nu}}{\partial \nu}\left(e^{-i \pi / 4} x\right)\right]\right\} .
\end{aligned}
$$

Similarly, subtracting (39) and (40), and taking into account the property

$$
\operatorname{Im} z+\operatorname{Re} z=\sqrt{2} \operatorname{Re}\left(e^{-i \pi / 4} z\right),
$$

we have

$$
\begin{aligned}
& \int_{0}^{1} u^{\nu / 2}\left[\gamma+\log \left(1-u^{2}\right)\right] \operatorname{bei}_{\nu}(x u) d u \\
= & \frac{1}{\sqrt{2} x}\left\{\left[-\frac{\pi}{4}+\log \left(\frac{x}{2}\right)\right] \operatorname{ber}_{\nu+1}(x)+\left[\frac{\pi}{4}+\log \left(\frac{x}{2}\right)\right] \operatorname{bei}_{\nu+1}(x)\right. \\
& \left.+\sqrt{2} \operatorname{Re}\left[e^{i \pi(\nu-1 / 4)} \frac{\partial J_{\nu}}{\partial \nu}\left(e^{-i \pi / 4} x\right)\right]\right\}
\end{aligned}
$$


Considering now the following indefinite integrals [5, Eqn. 10.71.1],

$$
\begin{aligned}
\int u^{\nu+1} \operatorname{ber}_{\nu}(u) d u & =\frac{u^{\nu+1}}{\sqrt{2}}\left[\operatorname{bei}_{\nu+1}(x)-\operatorname{ber}_{\nu+1}(x)\right], \\
\int u^{\nu+1} \operatorname{bei}_{\nu}(u) d u & =-\frac{u^{\nu+1}}{\sqrt{2}}\left[\operatorname{bei}_{\nu+1}(x)+\operatorname{ber}_{\nu+1}(x)\right],
\end{aligned}
$$

we obtain (38) with $f_{\nu}=\operatorname{ber}_{\nu}$ and $g_{\nu}=$ bei $_{\nu}$ from (41) and (43); and (38) with $f_{\nu}=$ bei $_{\nu}$ and $g_{\nu}=$ ber $_{\nu}$ from (42) and (44), as we wanted to prove.

\section{Conclusions}

We have obtained closed-form expressions of the derivatives of the Kelvin functions with respect to the order in (19)-(22) for $\nu \in \mathbb{R}$. These expressions are based on recent closed-form expressions found in the literature for $\partial J_{\nu} / \partial \nu$ and $\partial K_{\nu} / \partial \nu$, i.e. (34)-(37).

On the one hand, unlike the order derivatives of the $\operatorname{ber}_{\nu}$ and bei $_{\nu}$ functions found in the literature, i.e. (7) and (8), the results given in (19) and (20) are much more simple. Moreover, the expressions obtained for negative orders given in (27) and (28), in combination with (35) and (37), allow us the evaluation of the order derivatives of the $\operatorname{ber}_{\nu}$ and bei $_{\nu}$ functions for negative integral orders. The latter cannot be performed with the formulas found in the literature, i.e. (9) and (10). Also, the derivatives of the $\mathrm{ker}_{\nu}$, and $\mathrm{kei}_{\nu}$ functions with respect to the order given in (21) and (22) for non-negative orders, and the ones given in (32) and (33) for negative orders, seem to be novel.

On the other hand, we have calculated the integrals given in (38). These integrals do not seem to be reported in closed-form in the literature. Finally, in the Appendix, we provide an alternative derivation of the integral representations of the ber and bei functions found in the literature.

Acknowledgements It is a pleasure to thank Prof. A. Apelblat for the literature and wise comments given to the author.

\section{A Integral representations}

Theorem 5 The following integral representations hold true:

$$
\begin{aligned}
\operatorname{ber}(x) & =\frac{2}{\pi} \int_{0}^{\pi / 2} \cosh \left(\frac{x \operatorname{sc} \theta}{\sqrt{2}}\right) \cos \left(\frac{x \operatorname{sc} \theta}{\sqrt{2}}\right) d u, \\
\operatorname{bei}(x) & =\frac{2}{\pi} \int_{0}^{\pi / 2} \sinh \left(\frac{x \operatorname{sc} \theta}{\sqrt{2}}\right) \sin \left(\frac{x \operatorname{sc} \theta}{\sqrt{2}}\right) d u,
\end{aligned}
$$

where $\operatorname{sc} \theta=\sin \theta$ or $\cos \theta$. 
Proof. Multiply by $1 / s$ the trigonometric identity,

$$
\cos A+\cos B=2 \cos \left(\frac{A+B}{2}\right) \cos \left(\frac{A-B}{2}\right),
$$

and take $A=a / s, B=b / s$ to arrive at

$$
\frac{1}{s} \cos \left(\frac{a}{s}\right)+\frac{1}{s} \cos \left(\frac{b}{s}\right)=2 \frac{1}{\sqrt{s}} \cos \left(\frac{a+b}{2 s}\right) \frac{1}{\sqrt{s}} \cos \left(\frac{a-b}{2 \sqrt{s}}\right) .
$$

Defining the following functions $f, g, f_{1}$ and $g_{1}$ via the Laplace transform as follows,

$$
\begin{aligned}
\mathcal{L}[f] & =\frac{1}{s} \cos \left(\frac{a}{s}\right), \\
\mathcal{L}[g] & =\frac{1}{s} \cos \left(\frac{b}{s}\right), \\
\mathcal{L}\left[f_{1}\right] & =\frac{1}{s} \cos \left(\frac{a+b}{s}\right), \\
\mathcal{L}\left[g_{1}\right] & =\frac{1}{s} \cos \left(\frac{a-b}{s}\right),
\end{aligned}
$$

and applying the Laplace anti-transform to (48), we obtain

$$
\begin{aligned}
f+g & =2 \int_{0}^{t} f_{1}(t-\tau) g_{1}(\tau) d \tau \\
& =2 \int_{0}^{t} f_{1}(\tau) g_{1}(t-\tau) d \tau
\end{aligned}
$$

where we have applied the convolution theorem of the Laplace transform [11, Eqn. 17.12.5]. Since, according to [12, Eqn. 2.4.1(2)\&(3)] we have

$$
\begin{aligned}
\mathcal{L}^{-1}\left[\frac{1}{s} \cos \left(\frac{\alpha}{s}\right)\right] & =\operatorname{ber}(2 \sqrt{\alpha t}) \\
\mathcal{L}^{-1}\left[\frac{1}{\sqrt{s}} \cos \left(\frac{\alpha}{s}\right)\right] & =\frac{1}{\sqrt{\pi t}} \cosh \sqrt{2 \alpha t} \cos \sqrt{2 \alpha t}
\end{aligned}
$$

we rewrite (49) as

$$
\begin{aligned}
& \operatorname{ber}(2 \sqrt{a t})+\operatorname{ber}(2 \sqrt{b t}) \\
&= \frac{2}{\pi} \int_{0}^{t} \frac{\cosh \sqrt{(a+b)(t-\tau)} \cos \sqrt{(a+b)(t-\tau)}}{\sqrt{\tau(t-\tau)}} \\
& \cosh \sqrt{(a-b) \tau} \cos \sqrt{(a-b) \tau} d \tau .
\end{aligned}
$$

Taking $a=b$ and performing the changes of variables $x=2 \sqrt{a t}$ and $\tau=$ $z^{2} /(4 a)$, (51) becomes

$$
\operatorname{ber}(x)=\frac{1}{\pi} \int_{0}^{x} \frac{\cosh \sqrt{\frac{x^{2}-z^{2}}{2}} \cos \sqrt{\frac{x^{2}-z^{2}}{2}}}{\sqrt{x^{2}-z^{2}}} d z .
$$


Finally, performing the change $z=x \sin \theta$, we arrive at (45) with $\operatorname{sc} \theta=\cos \theta$. To arrive at (45) with $\operatorname{sc} \theta=\sin \theta$ we have to take (50) instead of (49) in the above reasoning. To obtain (46), we can depart from the trigonometric identity

$$
\sin A+\sin B=2 \sin \left(\frac{A+B}{2}\right) \sin \left(\frac{A-B}{2}\right),
$$

and consider the following Laplace anti-transforms [12, Eqn. 2.4.1(2)\&(3)]

$$
\begin{aligned}
\mathcal{L}^{-1}\left[\frac{1}{s} \sin \left(\frac{\alpha}{s}\right)\right] & =\operatorname{bei}(2 \sqrt{\alpha t}) \\
\mathcal{L}^{-1}\left[\frac{1}{\sqrt{s}} \sin \left(\frac{\alpha}{s}\right)\right] & =\frac{1}{\sqrt{\pi t}} \sinh \sqrt{2 \alpha t} \sin \sqrt{2 \alpha t} .
\end{aligned}
$$

Remark 6 It is worth noting that we can derive the integral representations (45) and (46) from the integral representations given in (3) and (4). For instance, taking $\nu=0$ and changing the argument $x \sqrt{2} \rightarrow x$ of the Kelvin function in (45), we have

$$
\text { ber }(x)=\frac{1}{\pi} \int_{0}^{\pi} \cos \left(\frac{x \sin t}{\sqrt{2}}\right) \cosh \left(\frac{x \sin t}{\sqrt{2}}\right) d t .
$$

Performing the change of variables $u=t-\pi / 2$ and taking into account the parity of the integrand, we arrive at

$$
\operatorname{ber}(x)=\frac{2}{\pi} \int_{0}^{\pi / 2} \cos \left(\frac{x \cos t}{\sqrt{2}}\right) \cosh \left(\frac{x \cos t}{\sqrt{2}}\right) d t,
$$

which is equivalent to (45) with $\operatorname{sc} \theta=\cos \theta$. Now, split (52) as

$$
\begin{aligned}
\operatorname{ber}(x)= & \frac{1}{\pi} \int_{0}^{\pi / 2} \cos \left(\frac{x \sin t}{\sqrt{2}}\right) \cosh \left(\frac{x \sin t}{\sqrt{2}}\right) d t \\
& +\frac{1}{\pi} \int_{\pi / 2}^{\pi} \cos \left(\frac{x \sin t}{\sqrt{2}}\right) \cosh \left(\frac{x \sin t}{\sqrt{2}}\right) d t
\end{aligned}
$$

and perform in (54) the change of variables $u=t-\pi / 2$, taking into account (53), to arrive at

$$
\operatorname{ber}(x)=\frac{1}{\pi} \int_{0}^{\pi / 2} \cos \left(\frac{x \sin t}{\sqrt{2}}\right) \cosh \left(\frac{x \sin t}{\sqrt{2}}\right) d t+\frac{1}{2} \operatorname{ber}(x),
$$

which is equivalent to 455) with $\operatorname{sc} \theta=\sin \theta$. For the integral representation of the bei function, we can perform a similar derivation. 


\section{References}

[1] Thomson W. Ether, electricity, and ponderable matter. In: Mathematical and Physical Papers. Cambridge: Cambridge University Press; 2011. p. 484-515.

[2] Russell A. The effective resistance and inductance of a concentric main, and methods of computing the ber and bei and allied functions. Philos. Mag. 1909;17:524-552.

[3] McLachlan NW. Bessel Functions for Engineers. 2nd ed. Oxford: Clarendon Press; 1961.

[4] Whitehead CS. On the generalization of the functions ber $\mathrm{x}$, bei $\mathrm{x}$, ker $\mathrm{x}$, kei x. Quart. J. Pure Appl. Math. 1911;42:316-342.

[5] Olver FWJ, Lozier DW, Boisvert RF, Clark CW, editors. NIST Handbook of Mathematical Functions. New York (NY): Cambridge University Press; 2010.

[6] Apelblat A. Integral representation of Kelvin functions and their derivatives with respect to the order. J. Appl. Math. Phys. 1991;42:708-714.

[7] Schulz-DuBois EO. Integral relations among Bessel functions. Math. Comput. $1969 ; 23: 845-847$.

[8] Brychkov YA. Higher derivatives of the Bessel functions with respect to the order. Integr. Transf. Spec. F. 2016;27:566-577.

[9] Brychkov YA. Handbook of special functions: derivatives, integrals, series and other formulas. Boca Raton (FL): CRC press; 2008.

[10] González-Santander JL. Closed-form expressions for derivatives of Bessel functions with respect to the order. J. Math. Anal. Appl. Available from: https://doi.org/10.1016/j.jmaa.2018.06.043

[11] Gradsthteyn IS, Ryzhik IM. Table of integrals, series and products, seventh ed. New York (NY): Academic Press; 2007.

[12] Prudnikov AP, Brychov YA, Marichev OI. Integrals and Series, vol. 5: Inverse Laplace Transforms. New York (NY): Gordon Breach; 1986. 